\title{
Significant Major Bleeding in Hospitalized Patients with COVID-19 Receiving Thromboprophylaxis
}

\author{
Vicky Mai ${ }^{1,2}$ Sabine Mainbourg ${ }^{3,4}$ Boun Kim Tan ${ }^{4}$ \\ ${ }^{1}$ Institut universitaire de cardiologie et de pneumologie de Québec \\ Research Center, Université Laval, Quebec City, Canada \\ 2 Pulmonary Hypertension Research Group, Quebec, Cananda \\ ${ }^{3}$ Laboratoire de Biométrie et Biologie Evolutive, Univ Lyon, UMR 5558 , \\ Villeurbanne, France \\ ${ }^{4}$ Department of Internal and Vascular Medicine, Centre Hospitalier \\ Lyon Sud, Hospices Civils de Lyon, Pierre-Bénite, France \\ ${ }^{5}$ Groupe d'Etude Multidisciplinaire des Maladies Thrombotiques \\ (GEMMAT), Hospices Civils de Lyon, Pierre-Bénite, France \\ 6 Department of Medicine, Université Laval, Quebec City, Canada
}

Thromb Haemost 2021;121:1670-1673.

The assessment of the thrombotic and hemorrhagic risks is essential when initiating thromboprophylaxis for the prevention of venous thromboembolism (VTE). Tan et $\mathrm{al}^{1}$ confirmed the elevated rates of VTE in patients with coronavirus disease 2019 (COVID-19) with an overall VTE prevalence of $15 \%$, reaching $23 \%$ in the intensive care unit (ICU). However, fewer studies evaluated the risk of major bleeding (MB). This is of importance since many institutional protocols adopted intermediate/therapeutic thromboprophylaxis dose based on the elevated risk of VTE while current guidelines recommend the use of thromboprophylaxis at a prophylactic dose in all hospitalized COVID-19 patients. ${ }^{2,3}$ We therefore read with great interest the article of Patell et $\mathrm{al}^{4}$ reporting a trend in higher bleeding rate in therapeutic-dose anticoagulants compared with standard-dose prophylaxis (6.3 vs. $1.7 \%$; $p=0.083$ ), advocating for further studies to define more precisely the rate of MB and guide the optimal thromboprophylaxis dosing.

As part of a systematic review on the incidence of COVID19-related VTE (PROSPERO-CRD42020183842), ${ }^{1}$ we also evaluated $\mathrm{MB}$ occurrence in hospitalized patients for COVID-19. We searched MEDLINE, Embase, and Google Scholar (January 1 to September 30, 2020). We included studies presenting the following criteria: (1) cohort of $>10$ patients, (2) patients with COVID-19; (3) data reporting MB. B.K.T. and J.-C.L. independently reviewed titles and abstracts of all articles, as well as full texts for deciding in their inclusion. V.M. and J.-C.L. independently extracted relevant information from selected papers. Disagreements were resolved by consensus or by consulting a third reviewer (S.P.).

received

February 6, 2021

accepted after revision

April 7, 2021

published online

April 8, 2021
Jean-Christophe Lega $a^{3,4,5}$ Steeve Provencher ${ }^{1,2,6}$

Address for correspondence Steeve Provencher, MD, MSc, Pulmonary Hypertension Research Group, Institut universitaire de cardiologie et de pneumologie de Québec Research Center, Université Laval, 2725 Chemin Ste-Foy, Quebec City, G1V 4G5, Canada (e-mail: steeve.provencher@criucpq.ulaval.ca).

The primary outcome for this subanalysis was the rate of MB in patients with COVID-19. A MB event was considered when the definition used in the study was defined according to the International Society of Thrombosis and Haemostasis criteria $^{5}$ or its equivalent, thereby the definition of MB could have varied across studies. The risk of bias of the selected studies, using the Methodological Index for Non-Randomized Studies (MINORS) ${ }^{6}$ for observational studies, and the strength of the body evidence, according to the GRADE system, were evaluated independently by V.M. and S.P. Publication bias was evaluated by a funnel plot.

Overall weighted frequency of MB was analyzed using R (meta package version 4.8-2 for pooled prevalence, $R$ Language and Environment for Statistical Computing, Vienna, Austria). ${ }^{7}$ Relative risks (RRs) were estimated with a $95 \%$ confidence interval (CI). A $p$-value $<0.05$ was considered statistically significant. $I^{2}>50 \%$ was considered as substantial statistical heterogeneity. Subgroup analyses compared patients admitted to the ICU to those admitted in ICU + general ward, as well as patients receiving intermediate/ therapeutic dose versus no/standard dose using Review Manager (Version 5.3., Copenhagen: The Nordic Cochrane Centre, The Cochrane Collaboration, 2014). ${ }^{8}$ Meta-regressions were made to evaluate association between MB and ICU hospitalization and anticoagulation intensity, respectively (rma function, metafor package).

Seventeen studies (10,722 patients) were included in our subanalysis (-Table 1 ). Seven studies ${ }^{9-15}$ included only patients from the ICU, 6 studies ${ }^{16-21}$ included mixed cohorts (ICU + general ward), 1 study $^{22}$ included no patients from the (c) 2021. Thieme. All rights reserved. Georg Thieme Verlag KG,

Rüdigerstraße 14,

70469 Stuttgart, Germany
DOI https://doi.org/ 10.1055/a-1477-4027. ISSN 0340-6245. 


\begin{tabular}{|c|c|c|c|c|c|c|c|c|c|c|c|c|c|c|c|c|c|c|c|c|c|}
\hline 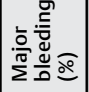 & $\begin{array}{l}\widehat{\varrho} \\
-\end{array}$ & $\underset{\infty}{\widehat{Q}}$ & $\underset{E}{E}$ & $\begin{array}{l}\widehat{\bar{d}} \\
\text { g }\end{array}$ & $\underset{\substack{\mathrm{J} \\
m}}{\mathrm{~J}}$ & $\widehat{\hat{m}}$ & $\underset{\sim}{\widehat{a}}$ & 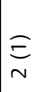 & 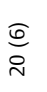 & $\begin{array}{l}\widehat{a} \\
\infty \\
\infty\end{array}$ & 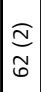 & $\begin{array}{l}\hat{\sigma} \\
\text { a } \\
m\end{array}$ & $\stackrel{0}{\stackrel{0}{\leftarrow}}$ & $\frac{\widehat{a}}{0}$ & 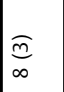 & $\underset{\infty}{\stackrel{0}{\sigma}}$ & $\frac{\widehat{n}}{a}$ & $\widehat{o}$ & $\underset{-}{\stackrel{\theta}{ \pm}}$ & $\frac{\Theta}{\sigma}$ & $\underset{-}{\Xi}$ \\
\hline 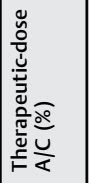 & 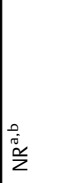 & 宸 & $=$ & nิ & $\stackrel{ \pm}{\sim}$ & 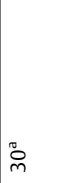 & \% & $\stackrel{\circ}{\circ}$ & $\stackrel{\infty}{\sim}$ & $\stackrel{\infty}{\sim}$ & $\stackrel{\infty}{\sim}$ & 离 & 尔 & $\stackrel{\infty}{\stackrel{\infty}{+}}$ & 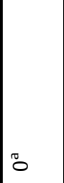 & $\tilde{\alpha}$ & 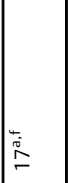 & $0_{0}^{\circ}$ & 0 & $\infty$ & ${ }^{\infty} \frac{\alpha}{2}$ \\
\hline 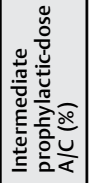 & 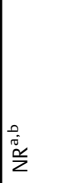 & 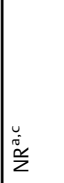 & 10 & 0 & 0 & 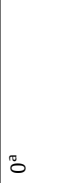 & ஜ̊ & : & $\wedge$ & $0_{0}^{\circ}$ & $\frac{a}{z}$ & $\stackrel{\sim}{\stackrel{\oplus}{*}}$ & $\stackrel{\oplus}{\check{m}}$ & ก̃ & \% & $\infty_{\infty}^{\infty}$ & 10 & 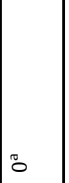 & $\stackrel{0}{\circ}$ & 岸 & $\stackrel{\infty}{2}$ \\
\hline 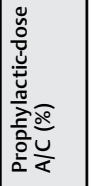 & "̊ & 正 & $\infty$ & f & $\stackrel{m}{r}$ & $\stackrel{0}{\circ}$ & ¿ & $\frac{\sigma}{\infty}$ & 5 & 范 & $\frac{\alpha}{z}$ & (ْ. & in & $\%$ & 車 & 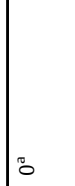 & $\frac{\pi}{\infty}$ & $\stackrel{\circ}{\circ}$ & 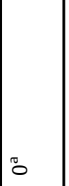 & $\frac{a}{z}$ & 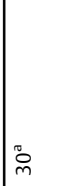 \\
\hline 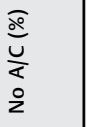 & $\stackrel{m}{m}$ & - & 0 & 0 & $m$ & 0 & 10 & Б & \% & $\stackrel{\pi}{\mathrm{m}}$ & $\sim$ & 离 & 俩 & 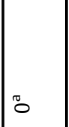 & \% & 10 & $\stackrel{D}{\sim}$ & 0 & \% & $\frac{a}{z}$ & ${ }^{\infty} \frac{\infty}{z}$ \\
\hline 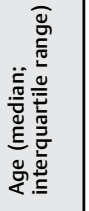 & 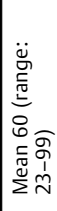 & 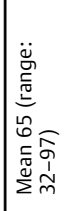 & 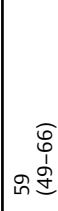 & 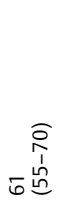 & $\widetilde{\sigma}$ & $\begin{array}{r}\widehat{i} \\
\hat{n} \\
\hat{0} \stackrel{0}{n}\end{array}$ & 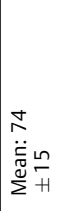 & 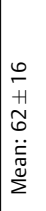 & 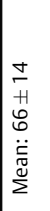 & $\begin{array}{l}\hat{n} \\
\hat{1} \\
\hat{n} \\
\tilde{n} \\
\tilde{n}\end{array}$ & $\frac{\alpha}{z}$ & 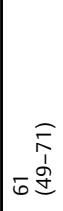 & 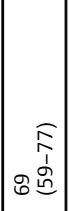 & 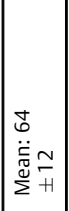 & 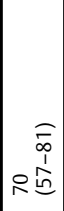 & 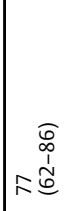 & 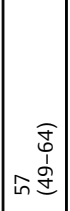 & 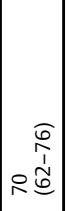 & 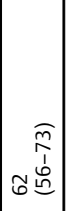 & 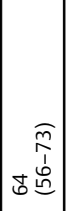 & 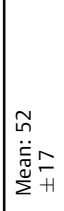 \\
\hline 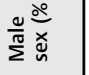 & ผิ & เి & ${ }^{n}$ & g & $\widetilde{\sigma}$ & $\bar{\infty}$ & $\stackrel{\infty}{\text { ก̊ }}$ & $\stackrel{\infty}{q}$ & in & in & $\frac{\mathscr{c}}{z}$ & in & g & 苗 & 节 & $\overline{6}$ & 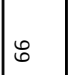 & $\frac{\pi}{\pi}$ & in & 音 & in \\
\hline 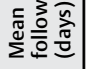 & $\frac{\tilde{c}}{z}$ & $\frac{x}{z}$ & $\stackrel{\infty}{\sim}$ & $\frac{\tilde{z}}{z}$ & $a$ & in & m & $\wedge$ & $\frac{\tilde{z}}{z}$ & $\frac{\stackrel{y}{z}}{2}$ & in & $\infty$ & $\sigma$ & m & m & m & থి & pे & 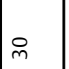 & $\frac{\tilde{c}}{z}$ & $\frac{\tilde{c}}{z}$ \\
\hline
\end{tabular}

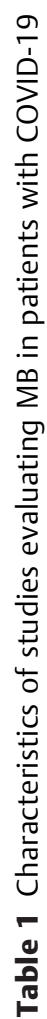

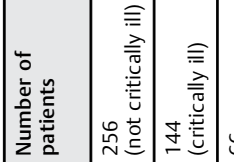

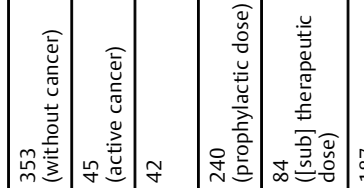

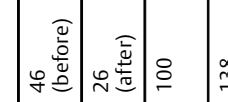
.5

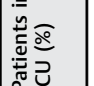

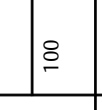

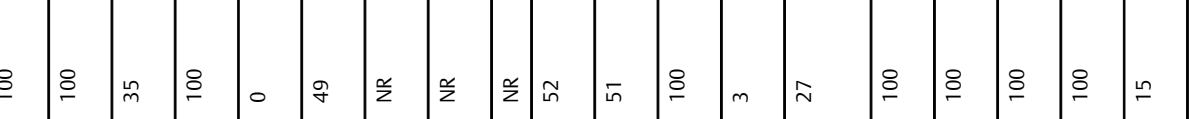

\begin{tabular}{|c|c|c|c|c|c|c|c|c|c|c|c|c|c|c|c|c|c|c|}
\hline 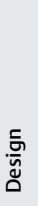 & 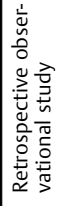 & 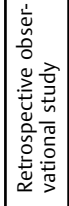 & 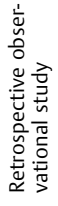 & 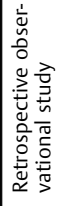 & 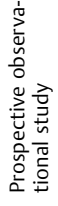 & 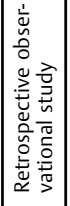 & 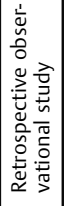 & 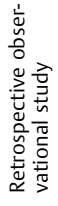 & 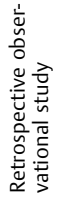 & $\frac{\mathscr{c}}{\mathrm{z}}$ & 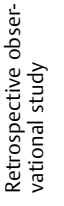 & 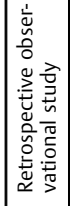 & 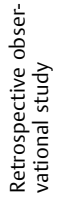 & 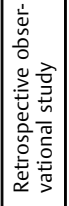 & 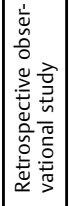 & 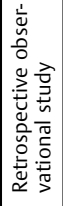 & 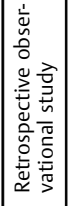 & 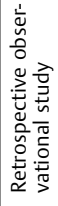 \\
\hline E & 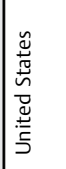 & 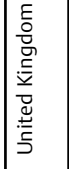 & $\begin{array}{l}\text { 岌 } \\
\text { 胥 }\end{array}$ & 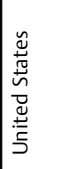 & $\begin{array}{l}\text { 岕 } \\
\text { 趈 }\end{array}$ & $\widehat{\widehat{N}}$ & 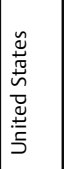 & 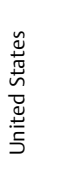 & 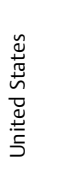 & 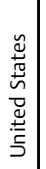 & 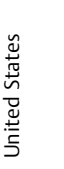 & $\widehat{\widehat{T}}$ & 产 & 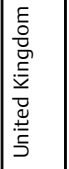 & 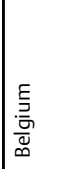 & & 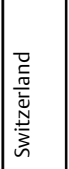 & 茎 \\
\hline 害 & 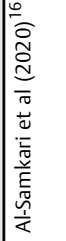 & 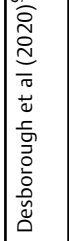 & 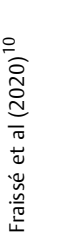 & 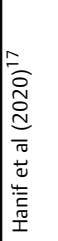 & 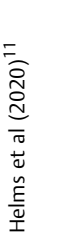 & 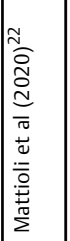 & 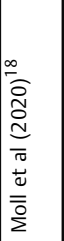 & 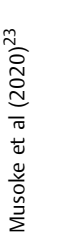 & 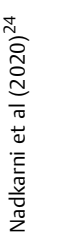 & 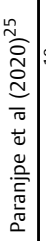 & 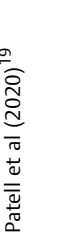 & 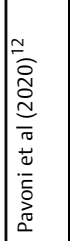 & 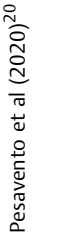 & 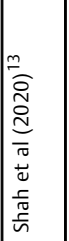 & 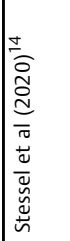 & & 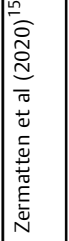 & 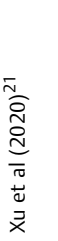 \\
\hline
\end{tabular}

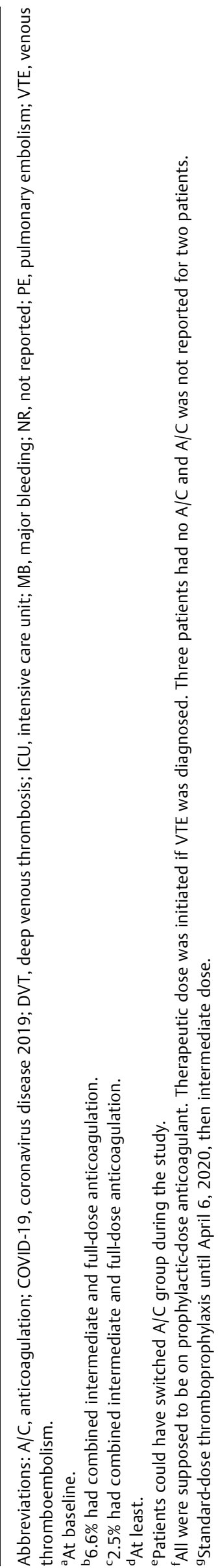




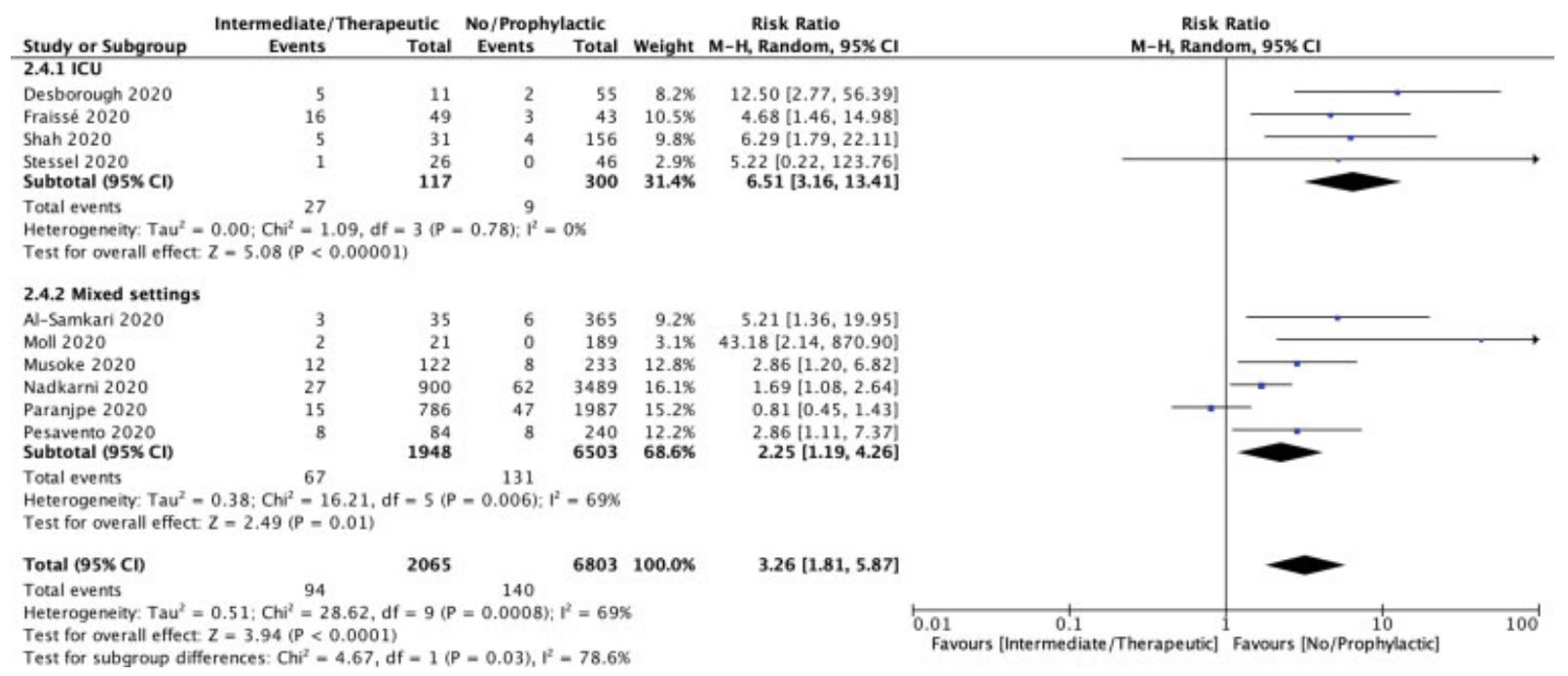

Fig. 1 Forest plot and relative risk for major bleeding (MB) in cohorts of patients hospitalized in the intensive care unit (ICU) and cohorts combining patients hospitalized in the ICU and general ward according to anticoagulation intensity. Amongst patients hospitalized in the ICU, the risk of MB was significantly increased in those receiving intermediate/therapeutic anticoagulation compared with no/standard prophylaxis at baseline. This association was also observed in cohorts combining patients hospitalized in the ICU and the general ward but to a lesser extend ( $p_{\text {interaction }}=0.03$ ).

ICU, and 3 studies ${ }^{23-25}$ did not report it. At baseline, the majority of the patients were on anticoagulation at a prophylactic dose in 10 studies, $9,11,13,14,16-20,23$ at an intermediate dose in 2 studies, ${ }^{12,22}$ and at a therapeutic dose in 1 study. $^{10}$

The overall weighted frequency of $\mathrm{MB}$ was $3.8 \%$ (95\% CI: $2.5-5.2 \% ; I^{2}=89 \% ; p_{\text {heterogeneity }}<0.01$ ). The funnel plot suggested publication bias. Meta-regression analyses revealed no significant association between the risk of $\mathrm{MB}$ and the proportion of patients hospitalized in the ICU $(p=0.60,14$ studies, 3,205 patients) or those receiving intermediate-/ therapeutic-dose anticoagulation ( $p=0.76,13$ studies, 3,105 patients). However, in studies including only patients hospitalized in the ICU, the risk of MB was significantly increased with intermediate/therapeutic anticoagulation versus no/standard prophylaxis (RR: 6.51; 95\% CI: 3.16-13.41; $p<0.001$ ) (-Fig. 1), occurring in 23.1\% (27/117) compared with $3.0 \%(9 / 300)$ in patients receiving no/standard prophylaxis. This association was also observed in mixed cohorts (ICU + general ward) of patients (RR: 2.25 ; 95\% CI: 1.19-4.26; $p=0.01$ ) (-Fig. 1), occurring in 3.4\% $(67 / 1,948)$ and $2.0 \%$ (131/6,503). The median MINORS score was 9 (range: 6-12). The strength of evidence was considered very low for MB.

The present meta-analysis reports an elevated overall MB weighted frequency of $3.8 \%$. To our knowledge, this is the largest cohort $(10,722$ patients) reporting the rate of $\mathrm{MB}$ in hospitalized patients with COVID-19. These data thus add to the article of Patell et $\mathrm{al}^{4}$ by providing up-to-date estimates on the risk of MB. While a high proportion of patients included in this meta-analysis were treated with a prophylactic dose, the observed MB rates were markedly higher than observed in non-COVID-19 patients hospitalized for acute VTE treated with therapeutic-dose low-molecularweight and unfractionated heparins, direct oral anticoagulants, and vitamin K antagonists, which resulted in MB in 1.5, $2.1,1.1$, and $1.7 \%$, respectively. ${ }^{26,27}$ The underlying mechanisms of increased $\mathrm{MB}$ remain elusive but may include
COVID-19-related endothelialitis, platelet dysfunction, and COVID-19-associated coagulopathy.

Importantly, the incidence of $\mathrm{MB}$ was highly variable across studies. Consistent with previous studies, we found no association between MB and ICU hospitalization ${ }^{28}$ or anticoagulation intensity, ${ }^{4}$ when analyzed individually. However, subgroup analyses, considered exploratory, suggested that ICU hospitalization and anticoagulation intensity may have synergetic effects, the risk of MB being markedly elevated in critically ill patients treated with intermediate-/ therapeutic-dose anticoagulation. This is further supported by a recent observational study documenting that therapeutic anticoagulation initiated within 48 hours following the admission to the ICU was associated with an increased risk of $\mathrm{MB}$, occurring in 60/384 (15.6\%) compared with 30/2,425 (1.2\%) of patients not initially anticoagulated (RR: $5.59 ; 95 \%$ CI: 4.68-6.69). ${ }^{29}$ These results may reflect the complex interplay between COVID-19 severity and anticoagulation intensity and may explain the recent pause in the recruitment of critically ill COVID-19 patients in ongoing anticoagulation trials.

We acknowledge that the present systematic review with meta-analysis presents some limitations. First, most of the studies presented data on baseline anticoagulation dosing, which may not reflect the number of patients receiving intermediate/therapeutic anticoagulation during the course of their disease. Second, confounding factors influencing bleeding (hepatic or renal insufficiency, antiplatelet therapy, and history of bleeding) could not be evaluated. Finally, our meta-regression failed to fully explain the heterogeneity associated with the risk of MB, whereas it could be partially explained by publication bias.

Our meta-analysis highlights the elevated risk of MB in hospitalized patients with COVID-19, regardless of the hospitalized setting and the anticoagulant dose. These results should be confirmed in prospective studies. Thus, the use of 
thromboprophylaxis at prophylactic dose should be maintained while awaiting for results of ongoing studies.

\section{Author Contributions}

V.M. contributed to study design, completed the literature search, data collection, data analysis, data interpretation, and drafted the first version of the manuscript. S.M. contributed to data interpretation and revised the manuscript. B.K.T. completed the literature search and data collection. J.-C.L. contributed to study design, literature search, data collection, data analysis, data interpretation, and revised the manuscript. S.P. contributed to study design, literature search, data analysis, data interpretation, and wrote and revised the manuscript.

Funding

None.

\section{Conflict of Interest}

V.M., S.M., B.K.T., and J.-C.L. have no conflict of interest. S. P. is clinician-scientist of the Fonds de Recherche en Santé du Québec and has received research grants from Actelion Pharmaceuticals, AstraZeneca, and Resverlogix outside of the submitted work.

\section{References}

1 Tan BK, Mainbourg S, Friggeri A, et al. Arterial and venous thromboembolism in COVID-19: a study-level meta-analysis. Thorax 2021;76(10):970-979

2 Moores LK, Tritschler T, Brosnahan S, et al. Prevention, diagnosis, and treatment of VTE in patients with coronavirus disease 2019: CHEST Guideline and Expert Panel Report. Chest 2020;158(03): 1143-1163

3 ASH. ASH Guidelines on Use of Anticoagulation in Patients with COVID-19.2020. Accessed May 2, 2021 at: https://www.hematology.org/education/clinicians/guidelines-and-quality-care/clinical-practice-guidelines/venous-thromboembolism-guidelines/ ashguidelines-on-use-of-anticoagulation-in-patients-with-covid-19

4 Patell R, Chiasakul T, Bauer E, Zwicker JI. Pharmacologic thromboprophylaxis and thrombosis in hospitalized patients with COVID19: a pooled analysis. Thromb Haemost 2021;121(01):76-85

5 Kaatz S, Ahmad D, Spyropoulos AC, Schulman SSubcommittee on Control of Anticoagulation. Definition of clinically relevant nonmajor bleeding in studies of anticoagulants in atrial fibrillation and venous thromboembolic disease in non-surgical patients: communication from the SSC of the ISTH. J Thromb Haemost 2015;13(11):2119-2126

6 Slim K, Nini E, Forestier D, Kwiatkowski F, Panis Y, Chipponi J. Methodological index for non-randomized studies (minors): development and validation of a new instrument. ANZ J Surg 2003;73(09):712-716

7 Viechtbauer W. Conducting meta-analyses in R with the metafor package. J Stat Softw 2010;36:1-48

8 Review Manager 5.3. Version 5.3.5. Copenhagen: The Nordic Cochrane Centre: The Cochrane Collaboration; 2014

9 Desborough MJR, Doyle AJ, Griffiths A, Retter A, Breen KA, Hunt BJ. Image-proven thromboembolism in patients with severe COVID19 in a tertiary critical care unit in the United Kingdom. Thromb Res 2020;193:1-4

10 Fraissé M, Logre E, Pajot O, Mentec H, Plantefève G, Contou D. Thrombotic and hemorrhagic events in critically ill COVID-19 patients: a French monocenter retrospective study. Crit Care 2020;24(01):275
11 Helms J, Tacquard C, Severac F, et al; CRICS TRIGGERSEP Group (Clinical Research in Intensive Care and Sepsis Trial Group for Global Evaluation and Research in Sepsis) High risk of thrombosis in patients with severe SARS-CoV-2 infection: a multicenter prospective cohort study. Intensive Care Med 2020;46(06): 1089-1098

12 Pavoni V, Gianesello L, Pazzi M, Stera C, Meconi T, Frigieri FC. Venous thromboembolism and bleeding in critically ill COVID-19 patients treated with higher than standard low molecular weight heparin doses and aspirin: A call to action. Thromb Res 2020; 196:313-317

13 Shah A, Donovan K, McHugh A, et al. Thrombotic and haemorrhagic complications in critically ill patients with COVID-19: a multicentre observational study. Crit Care 2020;24(01):561

14 Stessel B, Vanvuchelen C, Bruckers L, et al. Impact of implementation of an individualised thromboprophylaxis protocol in critically ill ICU patients with COVID-19: a longitudinal controlled before-after study. Thromb Res 2020;194:209-215

15 Zermatten MG, Pantet O, Gomez F, et al; COVID-19 Interdisciplinary Collaboration - COVIDIC initiative. Utility of D-dimers and intermediate-dose prophylaxis for venous thromboembolism in critically ill patients with COVID-19. Thromb Res 2020; 196:222-226

16 Al-Samkari H, Karp Leaf RS, Dzik WH, et al. COVID-19 and coagulation: bleeding and thrombotic manifestations of SARSCoV-2 infection. Blood 2020;136(04):489-500

17 Hanif A, Khan S, Mantri N, et al. Thrombotic complications and anticoagulation in COVID-19 pneumonia: a New York City hospital experience. Ann Hematol 2020;99(10):2323-2328

18 Moll M, Zon RL, Sylvester KW, et al. VTE in ICU patients with COVID-19. Chest 2020;158(05):2130-2135

19 Patell R, Bogue T, Bindal P, et al. Incidence of thrombosis and hemorrhage in hospitalized cancer patients with COVID-19. J Thromb Haemost 2020;18(09):2349-2357

20 Pesavento R, Ceccato D, Pasquetto G, et al. The hazard of (sub) therapeutic doses of anticoagulants in non-critically ill patients with Covid-19: the Padua province experience. J Thromb Haemost 2020;18(10):2629-2635

21 Xu JWL, Zhao L, Li Q et al. Risk assessment of venous thromboembolism and bleeding in COVID-19 patients. Res Square 2020. Doi: $10.21203 /$ rs.3.rs-18340/v1

22 Mattioli M, Benfaremo D, Mancini M, et al. Safety of intermediate dose of low molecular weight heparin in COVID-19 patients. J Thromb Thrombolysis 2020. Doi: 10.1007/s11239-020-02243-z

23 Musoke N, Lo KB, Albano J, et al. Anticoagulation and bleeding risk in patients with COVID-19. Thromb Res 2020;196:227-230

24 Nadkarni GN, Lala A, Bagiella E, et al. Anticoagulation, bleeding, mortality, and pathology in hospitalized patients with COVID-19. J Am Coll Cardiol 2020;76(16):1815-1826

25 Paranjpe I, Fuster V, Lala A, et al. Association of treatment dose anticoagulation with in-hospital survival among hospitalized patients with COVID-19. J Am Coll Cardiol 2020;76(01):122-124

26 Robertson L, Jones LE. Fixed dose subcutaneous low molecular weight heparins versus adjusted dose unfractionated heparin for the initial treatment of venous thromboembolism. Cochrane Database Syst Rev 2017;2:CD001100

27 Gómez-Outes A, Terleira-Fernández AI, Lecumberri R, Suárez-Gea ML, Vargas-Castrillón E. Direct oral anticoagulants in the treatment of acute venous thromboembolism: a systematic review and meta-analysis. Thromb Res 2014;134(04):774-782

28 Jiménez D, García-Sanchez A, Rali P, et al. Incidence of VTE and bleeding among hospitalized patients with coronavirus disease 2019: a systematic review and meta-analysis. Chest 2021;159 (03):1182-1196

29 Al-Samkari H, Gupta S, Leaf RK, et al;STOP-COVID-19 Investigators. Thrombosis, bleeding, and the observational effect of early therapeutic anticoagulation on survival in critically ill patients with COVID-19. Ann Intern Med 2021;174(05):622-632 